\title{
évacuation des déchets radioactifs en sites continentaux
}

\author{
par \\ Ph. Masure \\ B.R.G. M., Orléans
}

RESUME - Les projections très éloignées dans le temps qu'impose la gestion des déchets radioactifs à vie longue et les effets possibles du dégagement thermique auquel donnent lieu les déchets de haute activité, constituent des conditions inaccoutumées pour les spécialistes des nombreuses disciplines concernées par l'évacuation des déchets radioactifs en formations géologiques continentales.

La recherche de solutions fiables de confinement géologique ne peut se limiter à des projets de dépôts miniers dans des formations particulièrement favorables. Elle exige une connaissance des environnements géologique et écologique exceptionnellement précise et complète. Elle requiert une analyse prospective de leur évolution naturelle et de l'impact des actions humaines, totalement inaccoutumées de par l'échelle prévisionnelle qu'elle impose.

Se basant sur les résultats obtenus dans le cadre des programmes de recherche internationale en cours, 1'auteur propose un élargissement des réflexions portant sur le confinement géologique et visant à exploiter pleinement les connaissances scientifiques de la géosphère et les méthodes d'études disponibles.

\section{INTRODUCTION}

Les programmes de stockage souterrain de produits solides, liquides ou gazeux ont connu au cours de ces dernières années un développement remarquable. Que les raisons en soient technico-économiques, stratégiques de sécurité ou de gestion rationnelle de l'espace, il est prévisible que l'exploitation des vides naturels (pores, fractures, karsts), ou construits (cavités minées) comme volumes de stockage dans le sous-sol, va se poursuivre et même $s^{\prime a c c e ́ l e ́ r e r . ~}$

Les problèmes posés par l'évolution du milieu récepteur et son environnement au cours de l'exploitation du stockage ont donné lieu à des études scientifiques et techniques spécifiques (mécaniques, thermiques et hydrauliques) qui ont permis de mâttriser avec une fiabilité suffisante l'impact de tels projets sur l'environnement.

La situation est bien différente en matière d'évacuation de déchets de grande nocivité dans le sous-sol. La production de certains déchets industriels nocifs a atteint un niveau tel que leur dispersion dans le sol et les eaux n'est plus biologiquement satisfaisante. Leur transformation ou leur isolement. définitif de la biosphère s'impose.

Coutumiers et respectueux de normes de sûreté rigoureuses inhérentes à leur actifité, les spécialistes de l'industrie nucléaire sont les premiers - et probablement les seuls jusqu'ici - à avoir posé le problème des déchets de grande nocivité et à avoir initié les études scientifiques et techniques nécessaires.

RECHERCHES EN COURS SUR L'EVACUATION DES DECHETS RADIOACTIFS A VIE LONGUE EN FORMATIONS GEOLOGIQUES PROFONDES

Une partie des déchets solides produits au cours du cycle du combustible nucléaire renferme des radionucléides à vie longue (émetteurs alpha) qui sont actuellement isolés de l'environnement par stockage, en surface, dans l'attente de la mise en oeuvre de procédures d'évacuation. La nocivité de ces déchets de faible, moyenne ou haute activité persistera pour les générations futures durant des périodes qui s'étendent bien au-delà de l'avenir prévisible (plusieurs dizaines à plusieurs centaines de milliers d'années).

Les déchets de retraitement (:) recèlent des actinides, dont la très haute activité peut être considérée comme quasi-permanente. Ils contiennent également des produits de fission

(:) Déchets liquides calcinés et enrobés dans des verres boro-silicatés coulés dans des conteneurs métalliques 
dont la décroissance se traduit par un dégagement d'énergie thermique considérable au cours des premiers siècles qui suivent leur production (: $)$.

Les programmes de recherches relatifs à l'élimination de ces déchets de très haute activité, qui posent les problèmes scientifiques les plus complexes, se sont développés au cours des vingt dernières années aux USA et, plus récemment, dans les pays européens, le Canada et l'URSS. Parmi les concepts d'élimination variés qui ont été envisagés pour isoler ces déchets de la biosphère : évacuation dans l'espace extra-terrestre, destruction des radionucléides à vie longue par transmutation nucléaire, évacuation sur le fond des océans, évacuation sur le soubassement rocheux des calottes glaciaires ou dans les zones de subduction des plaques océaniques, évacuation profonde en formations géologiques stables, cette dernière alternative s'est vite imposée comme la plus accessible techniquement et la plus fiable scientifiquement, tout en paraissant économiquement raisonnable. Pour les mêmes raisons, le choix s'est porté en priorité sur le concept d'évacuation dans les formations continentales, de préférence à l'évacuation dans les formations sub-océaniques.

Le concept d'évacuation en formations géologiques profondes peut répondre a priori à un certain nombre d'exigences parmi lesquelles : inaccessibilité, stabilité à long terme, piégeage ou retardement du retour partiel des radionucléides jusqu'à la biosphère.

Les nappes d'eau souterraine ou l'eau interstitielle que l'on rencontre dans toute les roches de la partie superficielle de la croûte terrestre constitueront le principal facteur potentiel de libération et de transfert des radionucléides jusqu'à la biosphère. C'est pourquoi les formations géologiques retenues jusqu'ici comme milieux de confinement potentiels (sel, argiles et schistes, granites et gneiss, basaltes) ont été choisies pour leurs structures simples, leur abondance, leur faible perméabilité ou porosité en masse, leur conductivité thermique et/ou leur capacité de sorption élevée.

Les actions scientifiques et techniques internationales réalisées jusqu'ici ont porté sur la définition de stratégies possibles d'évacuation des déchets conditionnés en formations géologiques et sur la conception des installations de dépôt souterrain. Elles se sont attachées, à des degrés divers, à la recherche, la sélection et la caractérisation de sites géologiques potentiellement favorables au confinement des déchets radioactifs.

(:) $3.10^{12}$ Joules sont dégagés au cours du premier siècle par un conteneur de 1501 de déchets vitrifiés dont la concentration est de $12 \%$. Un site d'évacuation pourrait avoir une capacité allant jusqu'à quelques dizaines de milliers de conteneurs.
La connaissance des caractéristiques de libération des radionucléides par lixiviation des déchets conditionnés, puis des phénomènes de rétention (sorption-désorption) des principaux produits de fission (Sr, CS, Eu, I) et des actinides (diverses formes de $\mathrm{Pu}$, $\mathrm{Np}, \mathrm{Am}, \mathrm{Ra}$ ) et l'analyse détaillée des conditions hydrogéologiques dans les massifs rocheux ont fait I'objet des recherches les plus nombreuses et ont conduit à des programmes expérimentaux et de modélisation variés (écoulement hydraulique, phénomènes de transfert intégrant convection, dispersion et rétention). L'effort de caractérisation de l'évolution, à moyen et long terme, des barrières naturelles (milieu géologique récepteur) et artificielles (conteneurs métalliques et verres de conditionnement, matériaux de remplissage des cavités de dépôt) susceptibles de constituer le confinement des déchets radioactifs s'est heurté à de grandes difficultés. Il doit tenir compte, notamment, de l'interaction des différentes barrières avec les déchets et des conditions évolutives du milieu, liées aux effets du dégagement thermique et des radiations alpha.

L'évaluation de la sûreté à long terme de 1'isolation des radionucléides par le milieu géologique (étude d'impact) a également fait l'objet d'études nombreuses mais dispersées. Ces "analyses de sürete" comportent deux phases d'analyses complémentaires :

- L'étude des potentialitês de migration des éléments enfouis dans la géosphère depuis le dépôt d'évacuation jusqu'à la biosphère, dans le cadre de scénarios de défaillance représentatifs,

- L'évaluation des dommages causés dans la biosphère par la dispersion des radionucléides et des éléments chimiques stables provenant du dépôt (et éventuellement par des phénomènes particuliers liés à $1^{\prime}$ impact du dépôt sur la géosphère)

L'élaboration de scénarios de défaillance du confinement des déchets s'est faite suivant deux approches méthodologiques distinctes (probabiliste et déterministe). Sans discuter les mérites et limites respectifs des deux méthodes, on retiendra que l'approche probabiliste permet, par le développement d'analyses par arbres de défaillance du confinement (Fault Tree Analysis), de considérer de manière beaucoup plus complète et systématisée les situations pouvant conduire à la libération des déchets.

Dans le cadre des hypothèses de défaillance du confinement géologique les plus vraisemblables, la libération des déchets dans la biosphère est réduite et progressive. De plus, cette libération pourrait se produire à une époque très éloignée de la date d'évacuation des déchets. Des variations géographiques de la contamination radiologique sont probables, avec d'éventuels points de concentration localisés. Dans ces conditions, il est bien difficile de préciser des 
seuils dommageables et des risques pour des périodes de temps allant de plusieurs centaines à plusieurs milliers de générations humaines.

Au total, on peut considérer que les actions scientifiques et techniques développées jusqu'ici ont permis un fructueux rapprochement des spécialités concernées, qu'elles appartiennent au génie chimique, nucléaire ou minier, aux sciences de la terre ou à la radiobiologie. Toutefois, les actions entreprises nécessitent d'être poursuivies et un certain nombre de lacunes restent à combler pour s'assurer que tous les aspects du problème sont suffisamment appréhendés. L'effort de recherches correspondant risque d'être long et laborieux avant qu'un bilan scientifique définitif puisse être dressé.

Si cette situation est relativement compatible avec la cadence assez lente de production des déchets vitrifiés de très haute activité (déchets de retraitement), il en va tout autrement pour les déchets solides de baible et moyenne activite contamines par des produits à vie longue (: $)$ dont l'accumulation croissante des quantités produites nécessite que soient précisées de manière urgente des solutions sures d'isolement définitif de la biosphère. Aussi est-il indispensable que la recherche systématique, mais fractionnée, en cours soit accompagnée d'une réflexion opérationnelle globale portant sur le confinement géologique.

\section{PROPOSITIONS EN FAVEUR D'UNE REFLEXION OPE- RATIONNELLE SUR LE CONFINEMENT GEOLOGIQUE}

La communauté scientifique concernée par les problèmes d'évacuation de déchets à vie longue en formations géologiques ressent, dans une large mesure, les incertitudes bien connues des géologues, confrontés à des échelles de temps et d'espace sans commune mesure avec celles de l'expérimentation, à des lois de comportement mal appréhendées ou à des milieux mal définis. Pourtant, la métallogénie et l'étude des gisements d'hydrocarbures montrent que, dans des conditions particulières, certaines substances minérales ou organiques peuvent être piégées sous forme concentrée pendant des millions d'années.

Une réflexion opérationnelle sur le piégeage et le confinement géologique doit viser à exploiter pleinement les connaissances scientifiques acquises et les méthodes d'études disponibles, afin de disposer, dans un délai raisonnable, des éléments nécessaires pour juger des potentialités réelles de confinement à long terme présentées par certains sites géologiques, dans le cadre de stratégies d'évacuation adaptées. Cette réflexion doit se développer suivant deux thèmes majeurs :

(:) Boues de traitement des résidus liquides, gaines ou coques, déchets solides divers
- Comment limiter 1 'incertitude inhérente au milieu géologique et exploiter au mieux les potentialités naturelles de piégeage et confinement de la croute terrestre?

Comment optimiser les concepts d'évacuation retenus ?

\section{1 - Limitation des incertitudes actuelles}

La connaissance des équilibres internes des massifs s'est développée au cours des dernières décennies dans le cadre des projets de grands ouvrages de génie civil, d'exploitationshydrauliques souterraines et, plus récemment, de stockages souterrains. Les méthodes d'analyses, de mesures et de simulation mises en oeuvre avaient pour but de faire des prévisions de l'évolution des équilibres internes pour des périodes dépassant rarement quelques générations.

En butte aux incertitudes bien connues des spécialistes de génie géologique, les études de faisabilité de projets d'évacuation de déchets radioactifs à vie longue se heurtent en outre à des incertitudes jusqu'ici méconnues :

- celles qui sont liées à l'êchelle de temps "géologique" imposée par le problème, qui conduisent à tenir compte de situations, dimensions ou facteurs cinétiques jugés négligeables dans les raisonnements courants : amplitude de l'érosion superficielle à l'échelle du massif par exemple, influence des cycles climatiques lents sur les équilibres internes du milieu, effet à long terme des variations de température et des contraintes sur les conditions d'écoulement des fluides en milieux de très falble perméabilité, évaluation des risques d'intrusion accidentelle dans le dépôt souterrain par les générations futures, etc..

- celles qui sont liées au dégagement thermique des déchets de retraitement qui introduit une dimension nouvelle en matière d'impact des granđs ouvrages sur la géosphère, que ce soit des points de vue structural, minéralogique, géochimique, hydraulique ou mécanique. Après la découverte du "choc hydraulique" induit par les retenues de certains grands barrages, ou par l'injection massive de liquides dans certaines couches profondes, ne doit-on pas craindre un "choc thermique" ignoré jusqu'ici ?

Face aux incertitudes inhérentes aux opérations nouvelles d'aménagement, les programmes relatifs aux grands projets d'ouvrages souterrains (usines hydroélectriques souterraines, cavernes de stockage d'hydrocarbures, etc...) se déroulent généralement de manière pragmatique en mêlant, après une reconnaissance détaillée de sites sélectionnés, approche théorique et observations pratiques à grande échelle. La méthode appliquée consiste en une sélection et une adaptation progressive de divers projets en compétition aux conditions réelles du site, avec traitement éventuel du milieu naturel pour en corriger les défauts. 
- Appliquée au problème de l'évacuation des déchets radioactifs en formations géologiques continentales, cette méthode implique tout d'abord un nouvel effort de diversification des concepts de dépôt, facilité par la relative liberté de choix de sites favorables.

Les potentialités de piégeage consédérées jusqu'à présent dans les concepts de confinement ont été essentiellement d'ordre lithologique (granite, sel, argile). On a certainement trop négligé d'autres formes de piégeage rencontrées dans la nature et qui peuvent être d'origine structurale, stratigraphique, géochimique, capillaire, mécanique ou osmotique.

Les concentrations minérales non syngénétiques résultant du jeu des phénomènes géologiques dans des structures de piégeage singulières, il est important de compléter le concept trop général de formations géologiques potentiellement favorables aux confinements des radionucléides par le concept de contextes géologiques particulièrement favorables au piégeage des radionucléides, les déchets pouvant être considérés comme un minerai artificiel.

Cette nouvelle approche devrait inspirer de nombreuses variantes de confinement et d'adaptation des dépôts au milieu géologique en diversifiant les applications du concept multibarrière.

- Le concept de barrières artificielles autour des conteneurs de déchets conditionnés n'a fait référence, jusqu'ici, qu'à des matériaux inclus dans les puits forếs (barrières géochimiques). Il pourrait être enrichi, dans certains cas, par des solutions de traitement du milieu géologique entourant le dépôt en vue de limiter (ou éliminer) l'accès de l'eau souterraine dans le dépôt. A titre d'exemple, ceci pourrait être obtenu par injection de silice ou de précipités dans les fissures ou les pores, par drainage des abords du dépôt ou par l'utilisation de barrières capillaires. Certaines structures géologiques litées, judicieusement sélectionnées, pourraient également constituer une extension du concept multibarrière qui n'a pas été suffisamment évaluée et constituer ce que l'on pourrait appeler des barrières géologíques multiples.

- Enfin, si l'on veut être en mesure de faire des projections déterministes significatives de l'évolution à long terme des massifs rocheux, il est nécessaire de compléter et de préciser la connaissance des caractéristiques et du comportement du milieu géologique. Ainsi, les observations, mesures et expérimentations que permet l'exécution de puits et galeries expérimentales profondes doivent constituer 1'un des objectifs prioritaires de toute recherche dans ce domaine.

L'application des méthodes de génie géologique milite en faveur de la réalisation ultérieure d'installations pilotes expérimentales et reversibles (:), simulant 1'ouvrage final sur le lieu même des sites sélectionnés, en vue d'analyser l'impact du dépôt sur la géosphère, avec auscultation détaillée du massif et de l'ouvrage, et ce pendant une durée de l'ordre de plusieurs décennies.

\section{2 - optimisation des concepts de confinement}

L'objectif fondamental fixé aux analyses de sûreté est la vérification scientifique de la fiabilité du concept d'évacuation en formations géologiques. S'il est encore prématuré d'attendre des résultats complets d'analyse de risques exploitables dans le cadre d'une approche décisionnelle, les méthodes élaborées fournissent des outils qui peuvent être utilisés dès à présent en vue d'optimiser les concepts de confinement.

Si les développements d'analyse globale des performances de projets de confinement géologique se sont effectués dans le cadre d'approches probabilistes (fault tree analysis) ou déterministes (worst case analysis) dans le but de fournir des verdicts passifs, il semble que l'approche prospective fournisse un cadre plus adapté à une réflexion purement opérationnelle.

D'une manière générale, la prospective a pourbut de déterminer, à la lumière d'un faisceau représentatif de situations futures potentielles, les mesures à prendre immédiatement pour infléchir les tendances négatives. Son intérêt est de permettre de rapprocher, dans les scénarios cohérents, relations causales entre les différents facteurs du milieu et phénomènes aléatoires. Ces derniers peuvent être soit des produits du hasard (chute de météorites par exemple), soit des combinaisons accidentelles entre différentes séries de causes indépendantes les unes des autres.

Appliquée au cas particulier de l'évacuation des déchets rađioactifs, 1'approche prospective peut se subdiviser en trois actions principales qui conduisent à l'élaboration de scénarios simples et représentatifs :

- La première action est constituée par $\ell^{\prime}$ analyse historique de I'évolution des facteurs climatiques, biologiques et géologiques au cours du Quaternaire. Cette étude rétrospective du Quaternaire a pour objet de définir les tendances lourdes, les invariants dans le changement et les relations causales qui pourraient jouer sur l'évolution future du milieu naturel.

- Le deuxième domaine d'action concerne la caracterisation des milieux naturels et artificiels, de leur comportement et de leur évolution dans le temps. Il constitue le corps des recherches scientifiques actuelles. Il tient compte tout particulièrement de l'impact à long terme du dégagement thermique et de la migration des radionucléides sur l'environnement du dépôt.

(:) réversibles : permétant la récupération des conteneurs de déchets 
- Enfin, le troisième domaine d'action a trait à l'élaboration de scénarios d'êvolution possible de l'environnement géologique susceptible de favoriser la libération et la migration des radionucléides. La mise au point de tels scénarios peut être facilitée par les méthodes d'analyses par arbre de défaillance ou arbre d'événement.

Trois types de scénarios de base, građués dans la difficulté et la complexité, devraient être utilisés en vue de faciliter la comparaison des situations considérées. Il serait intéressant d'élaborer à ce propos des scénarios fixistes, des scénarios tendanciels et des scénarios catastrophistes.

- Les_scénarios fixistes supposent que l'équilíbre naturel actuel est immuable et que le milieu n'évolue que sous l'impact du dépôt. Cette hypothèse est vraisemblable à un horizon de quelques siècles.

- Les_scénarios tendanciels tiennent compte, en outre, des phénomeñes d'évolution naturelle tendancielle déterminés par 1 'analyse historique du milieu (climat, géologie, biologie). Ces hypothèses sont vraisemblables à un horizon allant de quelques millénaires à quelques dizaines de millénaires.

- Enfint_les_scénarios_catastrophistes compliquent les scénarios précédents, par la prise en compte d'événements catastrophiques à déroulement rapide, tels que secousses sismiques majeures, chute de météorites ou intrusion humaine accidentelle. Ils peuvent se substituer totalement aux autres scénarios pour les analyses de risques relatives à la phase opérationnelle de construction et d'évacuation des déchets, en tenant compte de phénomènes brutaux, comme l'inondation accidentelle du dépôt ou l'effondrement d'une galerie.

L'utilisation de I'approche prospective doit permettre d'atteindre à court terme deux objectifs bien précis :

- d'abord faire des comparaisons sur la fiabilité du confinement présenté par divers sites ou concepts d'évacuation en compétition. L'analyse des résultats, à l'interface géosphère-biosphère, des trois scénarios simples et reproductibles proposés facilitera cet objectif. Les multiples exercices d'analyse de risques effectués jusqu'ici par des équipes nationales compétentes n'ont pas permis, de par leur diversité, un tel rapprochement et une telle comparaison. Cette comparaison est pourtant indispensable, tant dans le domaine de la sûreté que dans le domaine des coûts.

- en second Iieu, faciliter la recherche des concepts d'évacuation les plus sûrs - c'està-dire les mieux adaptés - sur un site déterminé. Dans ce sens, les méthodes de simulation globale de systèmes et d'analyses de sensibilité fournissent l'outil nécessai- re de comparaison entre diverses options de confinement.

Ces méthodes devraient permettre de contrebalancer 1'effort de recherche systématique d'hypothèses de défaillance du confinement (fault tree analysis) qui a fait l'intérêt des approches probabilistes développées jusqu'ici en permettant :

i) de considérer à l'inverse les effets de certaines solutions de renforcement possible du confinement des déchets,

ii) de définir par effet rétroactif les performances minimales que les barrières construites devraient respecter, compte tenu des normes fixées pour la sûreté globale du système.

Il est bien évident que ces considérations doivent toujours être accompagnées par une analyse coût-bénéfices.

En résumé, l'approche prospective devrait permettre de juger de la pertinence de certaines variantes de concepts de dépôts (barrières artificielles complémentaires, traitement du rocher, etc..), ainsi que du coût et de la sûreté comparée de diverses stratégies de gestion et d'évacuation des déchets radioactifs. Si ces objectifs peuvent paraltre moins ambitieux que la démonstration scientifique de la sureté à long terme des projets d'évacuation élaborés, il n'en sont pas moins précieux au stade actuel des connaissances.

\section{CONCLUSION}

Les problèmes scientifiques et techniques posés par l'évacuation des déchets très nocifs dans le sous-sol, en vue de leur élimination définitive de la biosphère, sont bien différents de ceux que les spécialistes de stockage souterrain de substances récupérables ont à résoudre depuis une quinzaine d'années.

Malgré l'ampleur des recherches que nécessite la vérification scientifique de la fiabilité du concept de confinement géologique sur un site donné, l'existence de nombreuses concentrations minérales dans la géosphère prouve qu'il est légitime de chercher à tirex le meilleur parti des potentialités naturelles de confinement, dans le cadre de projets opérationnels adaptés et économiquement raisonnables. Il est pourtant curieux de constater que les programmes de recherche en cours sur l'évacuation des déchets radioactifs à vie longue ont porté tous leurs efforts initiaux sur la vérification toute théorique du respect de l'environnement pour les générations futures, en négligeant les enseignements apportés par 1'observation des contextes géologiques singuliers où peuvent être analysés "en vraie grandeur" les processus de piégeage et de confinement des éléments chimiques. 
L'analyse de la sûreté à long terme (pour l'homme et son environnement) présentée par les projets d'évacuation actuels a permis d'élaborer des méthodes d'approche nouvelles (analyses de risques) dont on a négligé, là aussi, l'intérêt qu'elles présentent pour optimiser les concepts d'évacuation envisagés.

Il semble donc nécessaire que ces connaissances soient rapidement exploitées, parallèlement à la poursuite de la recherche systématique entreprise, afin que soient précisées des concepts de confinement parfaitement fiables dans des délais brefs.

Devant l'accumulation croissante des déchets solides de faible et moyenne activité contaminés par des produits à vie longue, un effort de recherche et développement intensif est dorénavant crucial pour les pays à fort développement électro-nucléaire. Si cet effort n'était pas réalisé dans les années à venir, on pourrait craindre un véritable divorce entre les soucis des scientifiques et des organes de sûreté d'une part et les responsables de la gestion et de l'évacuation des déchets d'autre part.

Quoi qu'il en soit, il y a lieu de garder à l'esprit que le degré de raffinement ou de précision recherché dans certains développements théoriques peut être rendu caduque par les incertitudes inhérentes aux sciences de la terre, des matériaux, de l'environnement ou de l'homme et qu'ainsi une approche pragmatique des problèmes doit leur être constamment préférée pour être raisonnablement adaptée au stade actuel des connaissances.

Il serait grave, en particulier, que toute décision honnête et raisonnée soit bloquée par le malaise qu'apporte la prise de conscience toute nouvelle de l'impact à long terme des activités humaines sur 1 'environnement que nous préparons pour les générations futures.

Devrait-on stopper toute exploitation minière d'éléments chimiques nocifs puisqu'elle contribue à détruire leur piégeage naturel et à accélérer la contamination de l'environnement ? Doit-on proscrire toute utilisation du charbon, devant la pollution atmosphérique et les bouleversements climatiques que pourrait entrainer la croissance du taux de $\mathrm{CO}_{2}$ dans I'atmosphère? Il est important que la pratique des analyses de sûreté à long terme se multiplie dans tous les domaines de 1'activité humaine, afin que les comparaisons nécessaires à un choix raisonné soient possibles.
Nota : Une grande part des réflexions présentées dans ce document proviennent d'un travail effectué sous contrat pour la Commission des Communautés Européennes. Toutefois, les conclusions présentées sont strictement personnelles et n'engagent que l'auteur.

QUELQUES BASES D'INFORMATION SUR LES PROBLEMES DE GESTION ET EVACUATION DES DECHETS RADIOACTIFS.

- Rapport d'experts de 1'Agence pour 1'énergie nucléaire de $1^{\prime} O C D E$ : Objectifs, concepts et stratégies en matière de gestion des déchets radioactifs résultant des programmes nucléaires de puissance. Paris, Septembre 1977

- Agence Internationale de l'Energie Atomique. Agence de l'OCDE pour l'énergie nucléaire : Colloque international sur l'évacuation des déchets radioactifs dans le sol. Otaniemi, près Helsinki, 2-6 juillet 1979 .

- S. ORLOWSKI, R. SIMON. Première conférence européenne sur la gestion et le stockage des déchets radioactifs. Luxembourg, 20-23 mai 1980. Commission des Communautés Européennes. 